\title{
Elastic Constants of Nanometer Thick Diamond-like Carbon Films
}

Marco G. Beghi, Carlo E. Bottani, Andrea LiBassi, Rosanna Pastorelli, Brian K. Tanner ${ }^{1}$, Andrea C. Ferrari ${ }^{2}$ and John Robertson ${ }^{2}$

INFM and Nuclear Engineering Department, Politecnico di Milano, I-20133 Milan, Italy,

${ }^{1}$ Physics Department, University of Durham, Durham, DH1 3LE, UK

${ }^{2}$ Engineering Department, Cambridge University, Cambridge CB2 1PZ, UK

\section{ABSTRACT}

Carbon films of thickness down to 2 nanometers are necessary to achieve a storage density of $100 \mathrm{Gbit} / \mathrm{in}^{2}$ in magnetic hard disks. Reliable methods to measure the properties of these ultrathin films still have to be developed. We show for the first time that combining Surface Brillouin Scattering (SBS) and X-ray reflectivity measurements the elastic constants of such films can be obtained. Tetrahedral amorphous carbon films were deposited on silicon, by an $\mathrm{S}$ bend filtered cathodic vacuum arc, which provides a continuous coverage on large areas free of macroparticles. Films of thickness down to $2 \mathrm{~nm}$ and density of $\sim 3 \mathrm{~g} / \mathrm{cm}^{3}$ were produced and characterized. The dispersion relations of surface acoustic waves are measured by SBS for films of different thickness and for the bare substrate. Waves can be described by a continuum elastic model. Fitting of the dispersion relations, computed for given film properties, to the measured dispersion relations allows the derivation of the elastic constants. For a $8 \mathrm{~nm}$ thick film we find a Young's modulus E around $400 \mathrm{GPa}$, with a shear modulus $\mathrm{G}$ lying in the $130-210 \mathrm{GPa}$ interval. For a $4.5 \mathrm{~nm}$ thick film, $\mathrm{E}$ is around $240 \mathrm{GPa}$, with $\mathrm{G}$ lying in the $70-130 \mathrm{GPa}$ interval. Results for even thinner films become highly sensitive to the precision of the substrate properties, and indicate that the above values are lower bounds. We thus show that we can grow and characterize nanometer size tetrahedral amorphous carbon films, which maintain their density and mechanical properties down to the nm range.

\section{INTRODUCTION}

The storage density of magnetic hard disks is steadily increasing [1,2]. Technological developments are required to maintain the increasing trend. Namely, the distance between the recording head and the magnetic material has to be decreased, and this requires a reduction of the thickness of the protective coatings of the disk and the head. Carbon coatings are presently the standard choice: they provide protection against corrosion, grafting sites for the lubricant molecules and mechanical protection [1,2]. The continuity of the film is a crucial requisite. The achievement of a storage density of $100 \mathrm{Gbit} / \mathrm{in}^{2}$ requires a reduction of coating thickness down to 2-5 nanometers. The characterization of such thin films is still an open question. The elastic constants provide useful information: beside the direct characterization of the mechanical behavior, they give indirect indications on the quality and the continuity of the film. Although a film of few nanometers on a softer substrate cannot provide a strong mechanical protection [2], measurement of its elastic moduli provides however significant indications on film quality, including the fraction of $\mathrm{sp}^{3}$ bonding.

The stiffness of an amorphous, and therefore mechanically isotropic, film is fully characterized by two independent parameters, which are typically taken among Young's modulus E, shear modulus G, bulk modulus B, Poisson's ratio $v$ or among the elements $\mathrm{C}_{\mathrm{ij}}$ of the 
elastic tensor. Any two of them determine all the others. Their measurement for ultra-thin films is arduous. Indentation directly supplies the hardness, and is commonly adopted to measure also the elastic constants [3-5]. However for film thicknesses below $\sim 1 \mu \mathrm{m}$ the requisite of indentation depth significantly smaller than film thickness is met with difficulties, and, especially for hard films on softer substrates, the behaviour of the substrate can affect the results. For thicknesses of a few nanometers indentation is no longer viable. The elastic properties must be probed by other methods, namely exploiting acoustic waves.

Brillouin scattering, i.e. the scattering of an electromagnetic wave (a photon) by an acoustic wave (a long wavelength acoustic phonon) provides a convenient, contact less way to measure the spectrum of acoustic waves. Both bulk acoustic waves and surface acoustic waves (SAWs) can be measured. Brillouin scattering relies on thermally activated phonons, meaning that the acoustic amplitudes are small and that measurements are time consuming. However the exchanged wavevector is fully determined by the scattering geometry: surface phonons can be probed at wavelengths down to a quarter of a micron. Such acoustic wavelengths are significantly shorter than those probed by any other measurement technique. Since the acoustic velocities can be accurately computed as functions of the elastic moduli and the mass density, their measurement provides an access to the elastic properties. Brillouin scattering has proved able to measure the elastic properties of materials, exploiting either bulk acoustic waves [6] or Surface Brillouin Scattering (SBS), i.e. scattering by SAWs [7-12]. In particular in the case of layered structures, namely of a single isotropic layer on an anisotropic substrate, the elastic constants of films have been measured, for film thicknesses ranging from hundreds to tens of nanometers [10-12]. The measurement of film properties of thickness below $10 \mathrm{~nm}$ remained a challenge, which has been faced in this work.

\section{EXPERIMENT}

The tetrahedral amorphous carbon (ta-C) films were deposited using an S-Bend Filtered Cathodic Vacuum Arc (FCVA) with an integrated off plane double bend (S-Bend) magnetic filter. The deposition chamber was evacuated to $10^{-8}$ torr using a turbomolecular pump. ta-C films with particle area coverage of less than $0.01 \%$ and uniform cross section are consistently deposited with this system $[13,14]$. A series of films was grown for increasing deposition times defocusing the beam in order to achieve a small deposition rate and so to produce ultra-thin samples in a more controlled way. Thickness density and layering of these ultra-thin samples were derived by X-Ray reflectivity [15], Table I. Note that the structure of the ultra-thin S-bend FCVA films resembles that of thicker S-bend films [14], with a scaling of the bulk layer thickness, but not of the surface and interface layers (which are in the sub-nanometer range both in thick and thin films). Four samples are considered here: a bare silicon substrate, with a thin layer of native oxide due to exposition to air, and three carbon films.

SAW velocities of all the samples were measured by SBS: the specimen is illuminated by laser light and the spectrum of the scattered light is analyzed. Beside the strong peak of the elastically reflected light at frequency $\Omega$, the spectrum contains the doublet at frequencies $\Omega \pm \omega$, shifted by the SAW frequency $\omega$. The SAW wavevector $\mathrm{q}_{\|}$is selected by the scattering geometry (in backscattering, at incidence angle $\theta, \mathrm{q}_{\|}=2(2 \pi / \lambda) \sin \theta$, where $\lambda$ is the laser wavelength) and the SAW velocity $v=\omega / q_{\|}$is directly obtained. Measurements were performed with SAW propagation along the [100] direction on the (001) face of the Si substrate, at room temperature, in backscattering, with incidence angle $\theta$ from $30^{\circ}$ to $70^{\circ}$ (see Fig.1). The incident light is p-

W11.6.2 
Table I: Samples' properties

\begin{tabular}{|c|c|c|c|}
\hline Specimen & Film nature & $\begin{array}{c}\text { Film density } \\
\left(\mathrm{g} / \mathrm{cm}^{3}\right)\end{array}$ & $\begin{array}{c}\text { Film thickness } \\
(\mathrm{nm})\end{array}$ \\
\hline $\mathrm{A}$ & $\mathrm{SiO}_{\mathrm{x} \cdot}$ & -- & $\sim 2$ \\
$\mathrm{~B}$ & ta-C & 2.8 & 8 \\
$\mathrm{C}$ & ta-C & 2.8 & 4.5 \\
$\mathrm{D}$ & ta-C & 3.2 & 2.2 \\
\hline
\end{tabular}

polarised; the scattered light is collected without polarisation analysis, and analysed by a tandem $3+3$ pass high contrast interferometer of the Sandercock type. The light source was an Argon ion laser operating at $\lambda=514.5 \mathrm{~nm}$. The incident power on the specimen was around $100 \mathrm{~mW}$, focused into a spot of the order of $10^{3} \mu \mathrm{m}^{2}$; irradiation did not induce film modifications

\section{RESULTS}

Due to the low film thickness a single SAW is present, the Rayleigh wave modified by the presence of the film (Modified Rayleigh Wave, MRW). Its velocity can be computed [16] as function of the elastic constants and mass density of both the film and the substrate, of film thickness and on the wavevector $\mathrm{q}_{\|}$. It is thus possible to solve the inverse problem, i.e. the derivation of the elastic constants from measured SAW velocities [17]. The elastic constants and mass density of the anisotropic $\mathrm{Si}$ substrate are known $\left(\mathrm{C}_{11}=166 \mathrm{GPa}, \mathrm{C}_{12}=63.9 \mathrm{GPa}, \mathrm{C}_{44}=79.6\right.$ $\mathrm{GPa}, \rho=2.33 \mathrm{~g} / \mathrm{cm}^{3}[20]$ ), as well as the SAW wavevector and the film thickness and density. Thus the acoustic velocities remain functions of the unknown elastic constants of the film. It is

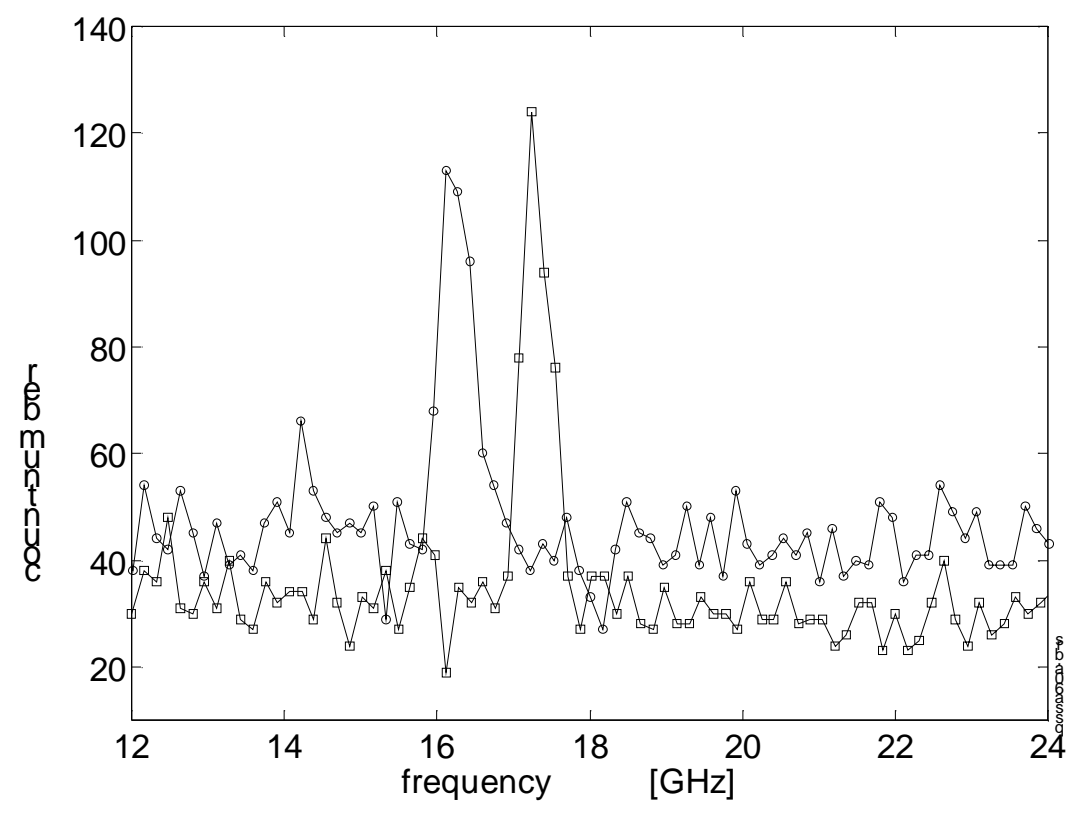

Figure 1. Portions of Brillouin spectra at $60^{\circ}$ incidence showing the peak due to modified Rayleigh wave, measured under identical conditions on specimens A (circles) and B (squares). 
known [11] that in this kind of films the properties can have a gradient in proximity of the outer surface and the inner interface but, to avoid exceeding complexities, the films are modeled as a single homogeneous equivalent film, with a sharp interface with the substrate.

It has been shown [18] that among the various elastic moduli that can represent the stiffness of an isotropic material the (E,G) pair is the most appropriate for the solution of the inverse problem. The velocities $\mathrm{v}_{\mathrm{c}}(\mathrm{E}, \mathrm{G})$ are computed for each value of $\mathrm{q}_{\|}$(i.e. for each incidence angle) at the nodes of a rectangular mesh. The couple $(\mathrm{E}, \mathrm{G})_{\text {film }}$ is obtained by a least squares fit of the computed velocities $\mathrm{v}_{\mathrm{c}}$ to the measured ones $\mathrm{v}_{\mathrm{m}}$ :

$$
R=\sum\left(\frac{v_{c}^{i}(E, G)-v_{m}^{i}}{\sigma_{e}^{i}}\right)^{2}=\min ,
$$

where $\sigma_{\mathrm{e}}{ }^{\mathrm{i}}$ are the variances of the corresponding $\mathrm{v}_{\mathrm{m}}{ }^{\mathrm{i}}$ due to the various uncertainties connected to the measurement (geometrical imperfections [19], statistical errors). The (E,G) couple which minimizes $R$ is the most probable solution of the inverse problem, and the regions corresponding to any fixed confidence level are also obtained.

It must be remembered that not all the (E,G) plane is physically meaningful. In this plane the $v=$ const lines are straight lines through the origin, and the $v=0.5$ and $v=0$ line delimit the triangle which is fully meaningful. The $v=0.5$ line is the limit for thermodynamic stability. When approaching or crossing this limit, the computed SAW velocities remain finite and continuous, but the bulk modulus B diverges. This means that if the confidence region falls close to the stability limit even a small region in the (E,G) plane, i.e. a good determination of both $\mathrm{E}$ and $\mathrm{G}$, still corresponds to a wide interval of $B$, which remains poorly determined. At the same time a physical plausibility limit can be imposed, considering only the part of the confidence region which corresponds to values of $\mathrm{B}$ below a pre-determined value. In this work the diamond value $\mathrm{B}=445 \mathrm{GPa}$ is taken a physically plausible upper limit. On the other side the $\mathrm{v}=0$ line does not mark a thermodynamic instability but, since negative values of Poisson's ratio can at most be found in extremely rare and peculiar cases, the $v=0$ line is taken as a physically plausible limit. Figure 2 shows the confidence regions found for specimen $\mathrm{B}$. The confidence regions cross the physically acceptable region, meaning that $v$ and $B$ remain essentially undetermined, while reasonably well defined intervals are found for $\mathrm{E}$ and $\mathrm{G}$ : $\mathrm{E}=350 \div 420 \mathrm{GPa}$ and $\mathrm{G}=130 \div 210$ $\mathrm{GPa}$. For specimen $\mathrm{C}$ the intervals are $\mathrm{E}=220 \div 260 \mathrm{GPa}$ and $\mathrm{G}=70 \div 130 \mathrm{GPa}, \nu$ and $\mathrm{B}$ remaining also undetermined. This is in agreement with the outcome of a sensitivity analysis study [18] which showed that $\mathrm{E}$ and $\mathrm{G}$ can be determined much better than $v$ and $\mathrm{B}$. The confidence regions must be seen as the real outcome of the measurements. Further resolution within the confidence regions has not a real significance. The obtained values of the elastic constants allow to fit the measured dispersion relations, as shown in Fig. 3. Figure 3 also explains the difficulty in obtaining the film elastic constants for specimens A and D, whose measured velocities are lower than those of a bare silicon substrate of nominal properties [20]. The fit for specimen A is in fact bad, and a better fit would require negligible film elastic constants and an unreasonably high mass density of silicon oxide. Also specimen D (carbon) seems to be acoustically slower, i.e. more compliant, than silicon. These findings suggest that in reality the actual properties of the silicon substrate are slightly different from the accepted values for pure silicon [20]. The stiffness of the substrate is slightly overestimated, implying that the film stiffness is underestimated. Due to the very small film thickness a very small overestimation of substrate stiffness is sufficient to lead to a significant underestimation of film stiffness.

W11.6.4 


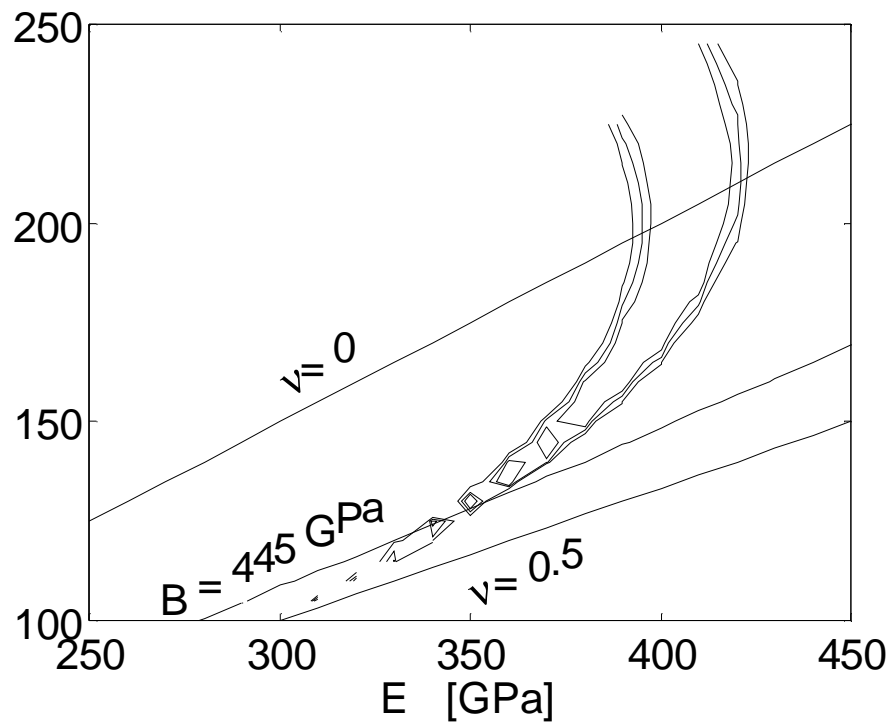

Figure $2.90 \%, 95 \%$ and $99 \%$ confidence regions in the $(\mathrm{E}, \mathrm{G})$ plane for specimen $\mathrm{B}$

\section{DISCUSSION AND CONCLUSIONS}

It has been shown that films only a very few nanometers thick exhibit dispersion relations of the modified Rayleigh wave that are measurably different. The analysis of these dispersion relations allows to measure the elastic constants of ta-C films, which have a significant stiffness also in films of 8 and 4 nanometres thick. For thinner films the analysis shows that a very precise characterization of the substrate is needed. The results for the thinnest films indicate that the values of the elastic constants found for the other films must be viewed as lower bounds.

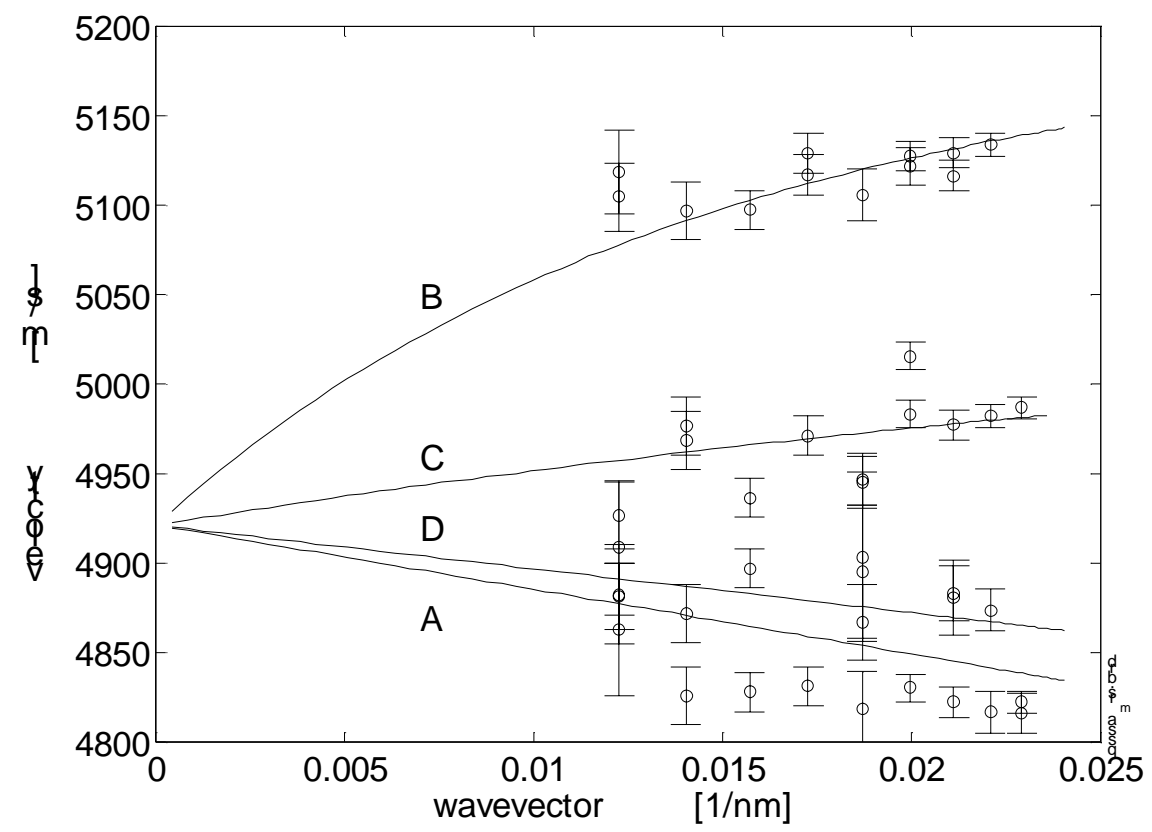

Figure 3. Measured velocities for the four specimens; dispersion relations computed with the film elastic moduli obtained from the best fit procedure, and accepted values of silicon properties [20]. The limit at null wavevector is the Rayleigh velocity of a bare silicon substrate. 


\section{ACKNOWLEDGEMENTS}

The authors thank K.B.K. Teo for assistance in sample preparation. A.C.F acknowledges funding from an E.U. TMR Marie Curie research fellowship and from Churchill College, Cambridge.

\section{REFERENCES}

1. P. R. Goglia, J. Berkowitz, J. Hoehn, A. Xidis and L. Stover, Diamond Relat. Mater. 10, 271277 (2001)

2. J. Robertson, Thin Solid Films 383, 81-88 (2001)

3. G.M. Pharr, D.L. Callahan, S.D. McAdams, T.Y. Tsui, S. Anders, J.W. Ager, I.G. Brown, C.S. Bhatia, S.R.P. Silva and J.Robertson, Appl. Phys. Lett., 68, 779 (1996)

4. J.A. Knapp, D.M. Follstaedt, S. M. Myers, J.C. Barbour and T. A. Friedmann, J. Appl. Phys. 85, 1460 (1999)

5. T. A. Friedmann, J. P. Sullivan, J.A. Knapp, D. R. Tallant, D.M. Follstaedt, D. L. Medlin and P. B. Mirkarimi, Appl. Phys. Lett., 71, 3820 (1997)

6. M. Grimsditch, in Handbook of elastic properties of solids, liquids and gases, Vol. 1, M. ed. by Levy et al. Academic Press / Harcourt Publishers Ltd., Sidcup, UK, 2000.

7. J. D. Comins, in Handbook of elastic properties of solids, liquids and gases, Vol. 1, ed. by M. Levy et al. Academic Press / Harcourt Publishers Ltd., Sidcup, UK, 2000.

8. P. Zinin P., M. H. Manghnani., S. Tlechev, V. Askarpour, O. Lefeuvre and A. Every, Phys. Rev. B, 602844 (1999).

9. M.G. Beghi, C.E. Bottani, P.M. Ossi, T. Lafford and B.K. Tanner, J. Appl. Phys., 81, 672 (1997), and references therein

10. A.C. Ferrari, J. Robertson, M.G. Beghi, C.E. Bottani, R. Ferulano and R. Pastorelli, Appl. Phys. Lett. 75, 1893 (1999)

11. A.C. Ferrari, J. Robertson, R. Pastorelli, M.G. Beghi and C.E. Bottani, Mat. Res. Soc., Symp. Proc. 594, 289 (2000)

12. M.G. Beghi, C.E. Bottani, and R. Pastorelli, in Mechanical Properties of Structural Films, ASTM STP 1413, ed. by C. Muhlstein and S. B. Brown, American Society for Testing and Materials, West Conshohocken, PA, 2001.

13. K.B.K. Teo, S.E. Rodil, J. T. H. Tsai, A. C. Ferrari, J. Robertson and W.I. Milne, J. Appl. Phys. 89, 3706 (2001)

14. A.C. Ferrari, A. LiBassi, B. K. Tanner, V. Stolojan, J. Yuan, L. M. Brown, S. E. Rodil, B. Kleinsorge, and J. Robertson, Phys. Rev. B 62, 11089 (2000).

15. B.K. Tanner, A. LiBassi, A.C. Ferrari and J. Robertson, these Proceedings.

16. G.W. Farnell and E.L. Adler, in Physical Acoustics, Vol. 9, ed. by W. P. Mason and R. N. Thurston, Academic, New York, 1972, p.35

17. R. Pastorelli, S. Tarantola, M.G. Beghi, C.E. Bottani, and A. Saltelli, in Mechanical Properties of Structural Films, ASTM STP 1413, ed. by C. Muhlstein and S. B. Brown, American Society for Testing and Materials, West Conshohocken, PA, 2001.

18. R. Pastorelli, S. Tarantola S., M.G. Beghi, C.E. Bottani and A. Saltelli, Surf. Sci. 468, 37 (2000).

19. R.P. Stoddart, Crowhurst J. C., Every A. G. and Comins J. D, J. Opt. Soc. of America B, 15, $2481(1998)$

20. H.J. McSkimin and P. Andreatch, J. Appl. Phys. 35, 3312 (1964) 